\title{
INDUCIBLE CLINDAMYCIN RESISTANCE IN ERYTHROMYCIN-RESISTANT STAPHYLOCOCCUS AUREUS
}

\author{
Vidya Shetty1, Jyothirlatha Bangera², Shubha Parameshwaran ${ }^{3}$
}

${ }^{1}$ Associate Professor, Department of Microbiology, KJ Somaiya Medical College and Hospital, Mumbai, Maharashtra. 2Professor and HOD, Department of Microbiology, KJ Somaiya Medical College and Hospital, Mumbai, Maharashtra. ${ }^{3}$ Ex-Professor and HOD, Department of Microbiology, KJ Somaiya Medical College and Hospital, Mumbai, Maharashtra.

\section{ABSTRACT}

\section{BACKGROUND}

The ERM gene product confers clindamycin resistance on Staphylococcus aureus. Clindamycin susceptible, erythromycin-resistant Staphylococcus aureus (Clindamycin-Erythromycin Discordant) may develop clindamycin resistance. Clindamycin is commonly used in the treatment of erythromycin-resistant S. aureus causing skin and soft infections. This led us to demonstrate a very simple method of detecting inducible resistance to Clindamycin in erythromycin-resistant S. aureus.

\section{MATERIALS AND METHODS}

This descriptive study of 94 erythromycin-resistant strains isolated from various clinical samples were subjected to D test as per CLSI guidelines.

\section{RESULTS}

Six (6.37\%) isolates showed constitutive MLSв phenotype, 60 (63.82\%) showed inducible MLSв phenotype, while 28 (29.78\%) gave MS phenotype. Inducible resistance and MS phenotype was found to be higher in MRSA.

\section{CONCLUSION}

Percentage of inducible resistance and constitutive clindamycin resistance were higher among MRSA than MSSA. Clinical Laboratories should report inducible clindamycin resistance in S. aureus. Study showed that D test should be used as a mandatory method in routine disc diffusion testing to detect inducible clindamycin resistance.

\section{KEYWORDS}

Clindamycin Resistance, MS Phenotype, Constitutive $\mathrm{MLS}_{\mathrm{B}}$ Phenotype, Inducible MLS ${ }_{\mathrm{B}}$ Phenotype.

HOW TO CITE THIS ARTICLE: Shetty V, Bangera J, Parameshwaran S. Inducible clindamycin resistance in erythromycin-resistant Staphylococcus aureus. J. Evolution Med. Dent. Sci. 2018;7(18):2202-2204, DOI: 10.14260/jemds/2018/495

\section{BACKGROUND}

Emergence of methicillin resistance in S. aureus has left us with very few therapeutic alternatives available to treat staphylococcal infections. The macrolide-lincosamide streptogramin $\mathrm{B}\left(\mathrm{MLS}_{\mathrm{B}}\right)$ family of antibiotics serve as one such alternative with Clindamycin being the preferred agent due its excellent Pharmacokinetic properties. ${ }^{1}$

The rapid emergence of community associated MRSA (CAMRSA) in the past several years in patients without obvious risk factors has complicated the management of both severe and localised staphylococcal infections. ${ }^{2}$

Clindamycin resistance in S. aureus can be either constitutive or inducible. The most common mechanism for such resistance is target site modification mediated by erm genes, which can be expressed either constitutively (Constitutive MLSB phenotype) or inducibly (inducible MLSB phenotype). Strains with inducible resistance to Clindamycin are difficult to detect on routine laboratory, as they appear erythromycin-resistant and Clindamycin-sensitive in vitro when not placed adjacent to each other. ${ }^{3}$

'Financial or Other Competing Interest': None.

Submission 19-03-2018, Peer Review 11-04-2018,

Acceptance 18-04-2018, Published 30-04-2018.

Corresponding Author:

Dr. Vidya Shetty,

Department of Microbiology,

KJ Somaiya Medical College and Hospital,

Everard Nagar, Sion, Mumbai-22, Maharashtra.

E-mail: vidyahshetty@yahoo.com

DOI: $10.14260 /$ jemds $/ 2018 / 495$

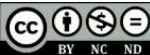

Clindamycin is frequently used to treat skin and bone infections because of its tolerability, cost, oral form and good tissue penetration. It may take time for a mutant strain to develop and the immune system may already have the infection. New agents active against gram-positive bacteria have been developed, so clindamycin may now be used less often. Finally, although a D test positive isolate may mutate on therapy, the mutation rate in clinical infections is unknown and may be rare.

\section{MATERIALS AND METHODS}

The descriptive study was conducted for a period of two years. A total of 94 isolates of S. aureus isolated from various clinical samples such as pus, wound swab, aspirates, blood and sterile fluids were tested. The isolates were first identified as S. aureus by standard biochemical techniques and then subjected to susceptibility testing by Kirby-Bauer's disc diffusion method on Mueller-Hinton agar plates as per CLSI guidelines. ${ }^{4}$

Those isolates which were erythromycin resistant were subjected to "D test" as per CLSI guidelines. Briefly erythromycin $(15 \mu \mathrm{g})$ disc was placed at a distance of $15 \mathrm{~mm}$ (Edge-to-Edge) from Clindamycin $(2 \mu \mathrm{g})$ disc on a MuellerHinton agar plate previously inoculated with 0.5 McFarland bacterial suspension. Following overnight incubation at $37^{\circ} \mathrm{C}$, flattening of zone (D shaped) around Clindamycin in the area between the two discs indicated inducible clindamycin resistance. 5 
Three different Phenotypes were Appreciated after testing and Interpreted as follows-

1. MS Phenotype- Staphylococcal isolates exhibiting resistance to erythromycin (Zone size $\leq 13 \mathrm{~mm}$ ), while sensitive to clindamycin (Zone size $\geq 21 \mathrm{~mm}$ ) and giving a circular zone of inhibition around clindamycin was labelled as having this phenotype.

2. Inducible $\mathrm{MLS}_{\mathrm{B}}$ Phenotype-Staphylococcal isolates showing resistance to erythromycin (Zone size $\leq 13$ $\mathrm{mm}$ ), while sensitive to clindamycin (Zone size $\geq 21 \mathrm{~mm}$ ) and giving a $\mathrm{D}$ shaped zone of inhibition around clindamycin with flattening erythromycin disc were labelled as having this phenotype.

3. Constitutive MLS $\mathrm{M}_{\mathrm{B}}$ Phenotype- This phenotype was labelled for those Staphylococcal isolates, which showed resistance to both erythromycin (Zone size $\leq 13 \mathrm{~mm}$ ) and clindamycin (Zone size $\geq 14 \mathrm{~mm}$ ) with circular shape of zone of inhibition if any around clindamycin.

\section{RESULTS}

A total of 94 strains of erythromycin-resistant strains were taken. These isolates were subjected to D test. Out of the 94 strains, $60(63.82 \%)$ were D test positive. 28 (29.78\%) strains showed circular zone of inhibition around Clindamycin suggesting MS phenotype.

Six isolates $(6.37 \%)$ were resistant to both erythromycin and clindamycin showing constitutive $\mathrm{MLS}_{\mathrm{B}}$ Phenotype.

The overall percentage resistance for all these phenotypes was as follows:

\begin{tabular}{|c|c|c|}
\hline Susceptibility Pattern & Phenotype & $\begin{array}{c}\text { Total No. of } \\
\text { Isolates (\%) }\end{array}$ \\
\hline ERY-S, CL-R & Constitutive & $6(6.37)$ \\
\hline ERY-R, CL-S (D Test Positive) & Inducible & $60(63.82)$ \\
\hline ERY-R, CL-S (D Test Negative) & MS & $28(29.78)$ \\
\hline \multicolumn{2}{|c|}{ Distribution of Isolates } \\
\hline
\end{tabular}

ERY- Erythromycin; CL- Clindamycin; S- Sensitive; RResistant; MS- MS Phenotype;

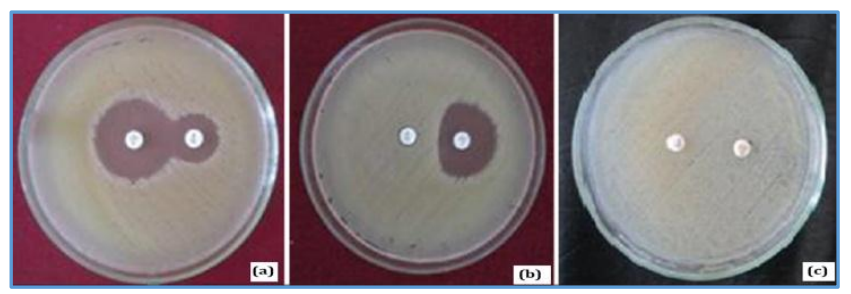

Disc Diffusion Test for Inducible Clindamycin Resistance a) Erythromycin-Resistant and Clindamycin Sensitive Staphylococcal Isolate with Circular Zone of Inhibition around Clindamycin suggestive of MS Phenotype

b) Erythromycin-Resistant and Clindamycin Sensitive Staphylococcal Isolate giving D-Shaped Zone of Inhibition around Clindamycin with Flattening towards Erythromycin Disc suggesting Inducible MLSB Phenotype c) Staphylococcal Isolate Resistant to both Erythromycin and Clindamycin suggestive of Constitutive MLSB Phenotype

\section{DISCUSSION}

The determination of antimicrobial susceptibility of a clinical isolate is often crucial for optimal antimicrobial therapy of infected patients. This is important considering the increase of resistance and the emergence of multidrug-resistant organisms. Clindamycin has become an excellent drug for some Staphylococcal infections, particularly skin and soft tissue infections and as an alternative in penicillin allergic patients. 6

Reporting S. aureus as susceptible to Clindamycin without checking for inducible resistance may result in institution of inappropriate Clindamycin therapy. On the other hand, negative result for inducible clindamycin resistance confirms Clindamycin susceptibility and provides a very good therapeutic option. ${ }^{7}$

In our study, six isolates (6.38\%) were resistant to Erythromycin and Clindamycin indicating Constitutive MLS Phenotype. This was in concordance with few studies by Deotale et al who observed $7.3 \%$ in MRSA strains. ${ }^{7}$ Angel et al study did not show any Constitutive resistance, which is contrary to our study. ${ }^{8}$

Inducible Clindamycin resistance was seen in 60 isolates $(63.82 \%)$. Another study from India showed high frequency of inducible resistance (63\%) in Erythromycin-Resistant Clindamycin sensitive isolates being $74 \%$ in MRSA and $45 \%$ in MSSA. ${ }^{9}$ On the contrary, Schreckenberger et al ${ }^{10}$ and Levin et al ${ }^{11}$ showed higher percentage of inducible resistance in MSSA as compared to MRSA.

Our study showed high percentage of Inducible Clindamycin resistance (63.82\%). Prabhu et al study showed $20(37.52 \%)$ isolates tested positive for Inducible Clindamycin resistance by D test, out of which 18 (16.66\%) were shown to have Constitutive Clindamycin resistance and $16(29.62 \%)$ showed true sensitivity to Clindamycin (MS Phenotype). ${ }^{3}$

The true sensitivity to Clindamycin can only be judged after performing D test on the erythromycin-resistant isolates. The prevalence of inducible clindamycin resistance may vary from hospital to hospital.

Use of D test in a routine laboratory will enable in guiding the clinicians regarding judicious use of Clindamycin in soft and soft tissue infections; as Clindamycin is not a suitable drug for $\mathrm{D}$ test positive isolates, while it can definitely prove to be a drug of choice in case of $\mathrm{D}$ test negative isolates.

A positive $\mathrm{D}$ test indicates the presence of macrolide inducible resistance to clindamycin produced by an inducible methylase that alters the common ribosomal binding site for macrolides, clindamycin and Group B streptogramins. For serious infections such as sepsis, pneumonia or other invasive S. aureus infections, even the small risk of emergence of resistance to clindamycin as indicated by a positive D test should lead to avoidance of use of Clindamycin. ${ }^{12}$

In the light of the restricted range of antibiotics available for the treatment of Methicillin-resistant staphylococcal infections and the known limitations of vancomycin, clindamycin should be considered for the management of serious soft tissue infections with methicillin-resistant staphylococci that are sensitive to clindamycin. ${ }^{13}$ The true sensitivity to clindamycin can only be judged after performing $\mathrm{D}$ test on erythromycin-resistant isolates. The prevalence of inducible clindamycin resistance may vary from hospital to hospital. 


\section{CONCLUSION}

D test can be used as a simple auxiliary and reliable method to delineate inducible and constitutive Clindamycin resistance in routine clinical laboratories. Use of $\mathrm{D}$ test in a routine laboratory will enable us in guiding the clinicians regarding judicious use of clindamycin in skin and soft tissue infections: as clindamycin is not a suitable drug for D test positive isolates, while it can definitely prove to be a drug of choice in case of $\mathrm{D}$ test negative isolates.

\section{REFERENCES}

[1] Fiebelkorn KR, Crawford SA, McElmeel ML, et al. Practical disc diffusion method for detection of inducible clindamycin resistance in staphylococcus aureus and coagulase negative staphylococci. J Clin Microbiol 2003;41(10):4740-4.

[2] Herold BC, Immergluck LC, Maranan MC, et al. Community acquired methicillin resistant Staphylococcus aureus in children with no identified predisposing risk. JAMA 1988;279(8):593-8.

[3] Prabhu K, Rao S, Rao V. Inducible clindamycin resistance in staphylococcus aureus isolated from clinical samples. J Lab Physicians 2011;3(1):25-7.

[4] Kloos WE, Banenman TL. Staphylococcus and micrococcus. Chapter 22. In Manual of clinical microbiology. $7^{\text {th }}$ edn. Murray PR, Baron EJ, Pfaller MA, et al. (eds). Washington DC: ASM Press, 1999:264-82.

[5] Clinical and Laboratory Standards Institute. Performance standards for Antimicrobial susceptibility Testing. $26^{\text {th }}$ edition, 2016:M100S.

[6] Drinkovic D, Fuller ER, Shore KP, et al. Clindamycin treatment of Staphylococcus aureus expressing inducible clindamycin resistance. J Antimicrob Chemother 2001;48(2):315-6.
[7] Deotale V, Mendiratta DK, Raut U, et al. Inducible clindamycin resistance in staphylococcus aureus isolated from clinical samples. Indian J Med Microbiol 2010;28(2):124-6.

[8] Angel MR, Balaji V, Prakash J, et al. Prevalence of inducible clindamycin resistance in gram positive organisms in a tertiary care centre. Indian J Med Microbiol 2008;26(3):262-4.

[9] Ajantha GS, Kulkarni RD, Shetty J, et al. Phenotype detection of inducible clindamycin resistance amongst staphylococcus aureus isolates by using lower limit of recommended inter-disk distance. Indian J Pathol Microbiol 2008;51(3):376-8.

[10] Schreckenberger PC, Ilendo E, Ristow KL. Incidence of constitutive and inducible clindamycin resistance in staphylococcus aureus and coagulase negative staphylococci in a community and a tertiary care hospital. J Clin Microbiol 2004;42(6):2777-9.

[11] Levin TP, Suh B, Axelrod P, et al. Potential clindamycin resistance in clindamycin susceptible, erythromycin resistant staphylococcus aureus: report of a clinical failure. Antimicrob Agents Chemother 2005;49(3):1222-4.

[12] Woods CR. Macrolide-inducible resistance to clindamycin and the $\mathrm{D}$ test. Pediatr Infect Dis J 2009;28(12):1115-8.

[13] Rao GG. Should clindamycin be used in treatment of patients with infections caused by erythromycin resistant staphylococci? J Antimicrob Chemother 2000;45(5):715. 